\title{
ENSAIOS EXPERIMENTAIS E SIMULAÇÃO NUMÉRICA DE VESTUÁRIO DE PROTEÇÃO INDIVIDUAL DE INCÊNDIO EXPOSTO AO CALOR RADIANTE
}

\section{RESUMO}

Vitor Pereira ${ }^{1}$

Paulo A. G. Piloto ${ }^{2}$

Khedouma Nechab Abdallah Benarous ${ }^{3}$

Neste artigo é apresentado um estudo de avaliação do comportamento de um conjunto muito significativo, de tecidos para proteção individual de segurança de bombeiros, quando submetidos a uma fonte de calor radiante. São apresentados ensaios experimentais desenvolvidos na Universidade de Tsinghua (TU, China) e no Instituto Politécnico de Bragança (IPB, Portugal). São apresentados os resultados de 84 ensaios experimentais no IPB, sendo 72 ensaios realizados para baixos níveis de fluxo de calor incidente $\left(5\left[\mathrm{~kW} / \mathrm{m}^{2}\right]\right)$ e 12 ensaios realizados para níveis médios de fluxo de calor incidente (20 $\left.\mathrm{kW} / \mathrm{m}^{2}\right]$ ). Estes ensaios permitiram determinar qual a melhor combinação de proteção para diferentes conjuntos de materiais com 3 camadas (camada exterior com 6 tecidos diferentes, barreira térmica com 4 tecidos diferentes e camada interior com 3 tecidos diferentes). A melhor combinação foi determinada pelo conjunto de material que apresentava menor densidade de fluxo de calor transmitido (TB3/BT2/F1 para baixos níveis de densidade de fluxo radiante e TB1/BT2/F1 para níveis médios de densidade de fluxo radiante). É ainda apresentado um estudo numérico 2D, não linear transiente, para baixos e médios níveis de densidades de fluxo de calor incidente. $O$ modelo numérico foi validado com os ensaios realizados na Universidade de Tsinghua. Estes resultados permitiram concluir que existe uma relação não linear entre o fluxo de calor incidente e o fluxo de calor transmitido, tendo em consideração o estudo paramétrico.

Palavras-chave: Fogo; Calor radiante; Bombeiro; Equipamento de proteção.

\footnotetext{
${ }^{1}$ MSc. Student. Instituto Politécnico de Bragança. Portugal

${ }^{2}$ Coordinator Professor. Instituto Politécnico de Bragança. Portugal

${ }^{3}$ Associate Professor. Université Saad Dahlab de Blida 1. Blida. Algerie
} 


\title{
EXPERIMENTAL TESTS AND NUMERICAL SIMULATION OF FIRE FIGHTER CLOTHING EXPOSED TO RADIANT HEAT
}

\begin{abstract}
This paper presents the results of a very significant number of radiant heat tests on different fabrics assemblies for individual fire safety protection. Experimental tests developed at Tsinghua University (TU, China) and at the Polytechnic Institute of Bragança (IPB, Portugal) are presented. A total of 84 experimental tests were carried out at IPB, of which 72 were performed for low incident heat flux level $\left(5\left[\mathrm{~kW} / \mathrm{m}^{2}\right]\right)$ and 12 tests for medium incident heat flux level (20 $\left.\left[\mathrm{kW} / \mathrm{m}^{2}\right]\right)$. These tests allowed to determine the best combination of protection for different assemblies of materials with 3 layers (outer layer with 6 different fabrics, thermal barrier with 4 different fabrics and inner layer with 3 different fabrics). The best combination was determined by the assembly with the lowest transmitted heat flux density (TB3/BT2/F1 for low radiation level and TB1/BT2/F1 for the medium radiation level). A two-dimensional nonlinear transient numerical model for low and medium incident heat flux is also presented. The numerical model was validated with the experimental tests performed at Tsinghua University. These results led to the conclusion that there is a nonlinear relationship between the incident heat flux and the transmitted heat flux, based on the parametric study.
\end{abstract}

\section{INTRODUÇÃO}

O equipamento de proteção individual (EPI) contra incêndio dos bombeiros foi investigado, nos últimos anos em vários aspetos, com o objetivo 
de melhorar a segurança dos seus utilizadores. Os EPI's foram projetados para proteger os bombeiros de condições excessivas de calor e fogo, permitindo um período de tempo para uma missão de resgate, combate a incêndio ou retirada do contacto direto com a chama. Os tecidos dos EPI são projetados para acomodar um certo grau de conforto aos bombeiros quando expostos a condições de acidente tipo incêndio. A fonte de calor, a intensidade do fogo, o tempo de exposição e outras variáveis, afetam o desempenho da proteção do EPI. O EPI é um conjunto de múltiplas camadas composto por vários tipos de tecidos e não tecidos. Nestes conjuntos poderão ser utilizadas fibras convencionais (algodão. lã e viscose) e fibras de alto desempenho [aramida. polibenzimidazol e polibenzoxazol (PBO)] (SONG, WANG, el al 2018). O EPI dos bombeiros é tradicionalmente um tecido de multicamadas que contém uma camada externa, barreira contra humidade, revestimento térmico e, por vezes, uma camada interna. $O$ tecido da camada externa deve proteger os bombeiros das condições iniciais de incêndio ou das condições de incêndio generalizado de um compartimento. A camada externa precisa de ter resistência suficiente quando submetido à tração. à abrasão e ao corte. A fibra usada para a camada externa é geralmente selecionada a partir de uma fibra retardadora de chama de alto desempenho. $\mathrm{O}$ objetivo da barreira contra a humidade é proteger o lado interno de água em estado líquido e permitir a passagem de água em estado de vapor no sentido contrário. Os bombeiros serão protegidos da água quente, enquanto o suor pode ser vaporizado e dissipado para o lado externo. $O$ revestimento térmico pode ser constituído por um subconjunto de um tecido ou não tecido. com o objetivo de redução do fator de transmissão de calor. $O$ revestimento térmico é a parte mais crítica no conjunto dos tecidos. porque tem o maior impacto na proteção térmica.

Diferentes estudos têm sido realizados para testar o comportamento térmico dos tecidos e também para avaliar o efeito da densidade de fluxo de calor transmitido para a pele humana. Alice Stoll em 1971 (STOLL e CHIANTA 1971) apresentaram uma investigação experimental para determinar a 
evolução da temperatura na região dos tecidos e da pele, utilizando um manequim submetido a um fluxo de calor radiante. As temperaturas experimentais foram comparadas com as fórmulas analíticas desenvolvidas pelos autores, tendo sido apresentada uma boa concordância para o valor da temperatura por unidade de densidade de fluxo de calor incidente. O limite inferior para o valor de lesão na pele foi identificado como sendo de cerca de $44 \stackrel{\circ}{\circ}$. Esta temperatura refere-se à camada interna da pele, posicionada a 80 $\mu \mathrm{m}$ abaixo da superfície externa da pele. $O$ dano na pele ocorre 100 vezes mais rápido a $50 \stackrel{\circ}{\circ}$ e o tecido da pele é completamente destruído a $72{ }^{\circ} \mathrm{C}$. Sun et al. (SUN, et al. 2000) estudaram o desempenho da proteção radiante, a permeabilidade ao ar, a evaporação de vapor e a resistência térmica da roupa, em função das estruturas químicas e físicas dos tecidos. Os resultados indicam que quanto maior for a espessura ou mais pesado for o tecido, melhor é a proteção radiante. No mesmo ano de 2000, Lawson et al. (MELL e LAWSON, 2000) apresentaram um modelo unidimensional de transferência de calor para roupas de proteção de bombeiros, assumindo que os tecidos estavam secos e as temperaturas do tecido estavam abaixo do ponto de degradação térmica (fusão ou carbonização). O modelo incorpora um esquema de diferenças finitas para resolver a equação de energia nos domínios sólido e gás, aproximando as derivadas de primeira ordem, em vez das tradicionais derivadas de segunda ordem e utilizando um esquema incremental de avanço no tempo de RungeKutta. O modelo foi validado com resultados experimentais, sendo a principal diferença entre eles igual a $5{ }^{\circ} \mathrm{C}$ para as camadas mais interiores do conjunto de tecidos e $24{ }^{\circ} \mathrm{C}$ para a camada exterior. Em 2005. Kutlu et al. (KUTLU e CIRELI, 2005) concluem que os fatores que mais influenciam as classificações de desempenho de proteção térmica são a espessura e o tipo de fibra dos tecidos. Também investigaram a durabilidade do EPI, submetendo os tecidos a 10 ciclos de lavagem na máquina a $40 \stackrel{\circ}{ } \mathrm{C}$, tendo verificado uma diminuição na resistência ao rasgo dos tecidos, mas um aumento no desempenho de proteção térmica devido ao efeito aveludado, juntamente com o aumento da 
quantidade de ar retido entre as camadas das fibras. Lawson et al em 2010 (JAMES; LAWSON; MELL e PRASAD 2010) decidiram melhorar o modelo do ano 2000, introduzindo o parâmetro humidade no modelo dos tecidos e introduzindo uma base de dados de materiais com propriedades térmicas, utilizada para o fabrico roupas de proteção para bombeiros. O modelo incluiu as equações que governam a transferência de calor e massa (humidade), para condições de fluxo de calor radiante. A diferença máxima entre os resultados experimentais e os resultados numéricos foi de $6 \%$. Os resultados mostraram que a humidade nos tecidos tende a evaporar durante o aquecimento, sendo parte difundida e condensada em diferentes partes do tecido, dependendo da temperatura ambiente. Em 2012, Ghazy et al. (GHAZY e BERGSTROM, 2012) desenvolveram um modelo de volumes finitos para a transferência de calor transiente nas roupas de proteção dos bombeiros, usando uma análise mais sofisticada e apropriada para 0 ar aprisionado na estrutura dos tecidos. Os resultados numéricos indicaram que a influência do ar aprisionado na estrutura dos tecidos aumenta da zona exterior para a zona interior. Desde então, diferentes autores deram o seu contributo para a investigação do efeito da humidade e na durabilidade das roupas de proteção contra incêndios em relação aos ciclos de lavagem (ATALAY; BAHADIR e KALAOGLU, 2015), tendo verificado que este ciclos deterioram o desempenho térmico do conjunto. Outros autores em 2016 (TIAN; WANG e LI, 2016) desenvolveram um modelo 3D, baseado na dinâmica de fluidos computacional, para simular o campo de temperatura em cada camada dos tecidos aplicados num manequim. Os resultados numéricos foram validados com resultados experimentais em todos os locais de medição. Os modelos incluíram diferentes valores de folgas de ar, sendo que a espessura de $6.35 \mathrm{~mm}$ foi responsável por uma resistência mais eficiente, quando comparado com o efeito térmico de um tecido de camada simples. Neste sistema de roupas de várias camadas, a camada da barreira contra humidade foi identificada como a camada mais eficaz na proteção térmica durante o incêndio. Em 2018, S. Dahamni et al (DAHAMNI, et al. 2018) 
apresentaram os resultados de uma simulação numérica para previsão do fluxo térmico recebido pela roupa de proteção de um bombeiro, tentando avaliar o efeito das condições de ventilação no compartimento confinado. Apesar da importância dos dispositivos de ventilação mecânica, as taxas de fluxo de ventilação podem levar a enormes fluxos de calor que podem afetar severamente os EPIs.

O desempenho térmico, a resistência mecânica e a durabilidade são características importantes que devem ser avaliadas sob condições de acidente. Esta investigação trata da análise do desempenho térmico de diferentes tecidos num conjunto multicamadas. Dois tecidos diferentes foram testados experimentalmente na Universidade de Tsinghua (TU, China) usando baixos fluxos de calor radiante, enquanto treze tecidos diferentes foram testados experimentalmente no Instituto Politécnico de Bragança, utilizando níveis baixos e médios de fluxos de calor radiante. Ambos os resultados experimentais são apresentados, com base no método de ensaio $B$ para avaliação dos conjuntos de materiais quando expostos a uma fonte de calor radiante NP EN 6942 (CEN - European Committee for Standardization 2005), usando um calorímetro de cone semelhante ao utilizado na norma EN ISO 13927 (CEN - European Committee for Standardization 2003). As amostras foram posicionadas na superfície de uma placa de cobre, diretamente expostas a um nível específico de fluxo de calor radiante. Os tempos para o aumento da temperatura em $12\left[{ }^{\circ} \mathrm{C}\right]$ e $24\left[{ }^{\circ} \mathrm{C}\right]$, em relação ao valor da temperatura inicial, são registados no calorímetro e expressos como índices de transferência de calor radiante. A percentagem do fator de transmissão de calor também é determinada.

Esta investigação também apresenta uma validação numérica dos testes experimentais desenvolvidos na Universidade de Tsinghua, para avaliar o desempenho térmico das amostras de tecidos dos EPIs. Este modelo numérico 2D resolve a equação da energia, utilizando um modelo de comportamento não linear material sob baixa radiação térmica (CEN - European Committee for 
Standardization 2005). A validação numérica é baseada no teste de ensaio do calorímetro de cone. em escala reduzida, expondo o conjunto dos tecidos a uma baixa radiação térmica de 2, 5 e $10\left[\mathrm{~kW} / \mathrm{m}^{2}\right]$, seguindo os procedimentos da NP EN ISO 6942 (CEN - European Committee for Standardization 2005). Os resultados numéricos concordam, razoavelmente bem, com os resultados experimentais, em particular para as temperaturas T1, T2 e T3, permitindo aos autores desenvolver um estudo paramétrico para diferentes valores de densidade de fluxo de calor radiante 1, 3, 5, 7 e $20\left[\mathrm{~kW} / \mathrm{m}^{2}\right]$.

\section{ENSAIOS DE RADIAÇÃO}

O teste de radiação foi realizado com um calorímetro e um dispositivo para medição da temperatura em cada camada do conjunto de tecidos e na superfície não exposta da placa de cobre, usando uma frequência de aquisição de dados de $10 \mathrm{~Hz}$. A Figura 1 representa a configuração usada para os ensaios realizados no Instituto Politécnico de Bragança (IPB). Esta configuração foi semelhante à usada na Universidade de Tsinghua (TU), ver Figura 1. Foram utilizados termopares tipo $\mathrm{K}$ para medir as temperaturas na superfície superior de cada tecido. Além disso, foi efetuada a gravação de imagens térmicas de infravermelhos da superfície exposta. A placa de cobre possui $99 \%$ de pureza e tem $1.6 \mathrm{~mm}$ de espessura, sendo dobrada na direção mais longa de maneira a formar um arco com um raio de $130 \mathrm{~mm}$. Esta placa está localizada num bloco de montagem quadrado de $90 \mathrm{~mm}$, feito de material isolante térmico não combustível, livre de asbestos amianto. Os ensaios poderão ser realizados para densidades de fluxo de calor incidente divididos em três níveis diferentes: baixo, médio e alto. Esta investigação utilizou nível baixo de radiação térmica $\left(<10\left[\mathrm{~kW} / \mathrm{m}^{2}\right]\right)$ e o nível médio de radiação térmica (20 $\left.\left[\mathrm{kW} / \mathrm{m}^{2}\right]\right)$. O fluxo de calor foi calibrado com um sensor de fluxo de calor, levando em consideração a posição das amostras $(60 \mathrm{~mm}$ abaixo da parte inferior das resistências do calorímetro de cone). 
As amostras foram preparadas com $200 \mathrm{~mm}$ de comprimento e $100 \mathrm{~mm}$ de largura. costurados num dos lados mais pequeno. Foram utilizadas massas especiais colocadas nas arestas mais curtas das amostras para garantir que estas se encontravam esticadas e em contacto com a superfície de cobre. $O$ teste foi iniciado depois da placa de proteção móvel do calorímetro de cone ser retirada. Foi determinado o tempo $t_{12}$ para atingir um aumento de temperatura de $12\left[{ }^{\circ} \mathrm{C}\right]$ e o tempo $\mathrm{t}_{24}$ para atingir um aumento de temperatura de $\left.24{ }^{\circ} \mathrm{C}\right]$, em relação ao valor da temperatura média inicial na superfície não exposta da placa de cobre. Esta diferença também é necessária para determinar a densidade do fluxo de calor transmitido a partir da superfície inferior da chapa de cobre. A densidade do fluxo de calor transmitido, $Q_{c}$, é calculada de acordo com a Eq.1. assumindo o modelo de capacitância térmica global aplicado na placa de cobre.

$$
Q_{c}=\frac{M \cdot C_{p}}{A \cdot\left(t_{24}-t_{12}\right) / 12}
$$

M representa a massa da placa de cobre [kg], Cp representa o calor específico do cobre $[\mathrm{kJ} / \mathrm{kg} \mathrm{K}]$, A representa a área da placa de cobre $\left[\mathrm{m}^{2}\right]$ e o quociente $12 /\left(\mathrm{t}_{24}-\mathrm{t}_{12}\right)$ representa a taxa média do aumento da temperatura [으요.

O fator de transmissão de calor $\operatorname{TF}\left(Q_{0}\right)$ para o nível de densidade de fluxo de calor incidente $Q_{0}$ pode ser calculado pela Eq. 2.

$$
T F\left(Q_{0}\right)=\frac{Q_{c}}{Q_{0}}
$$




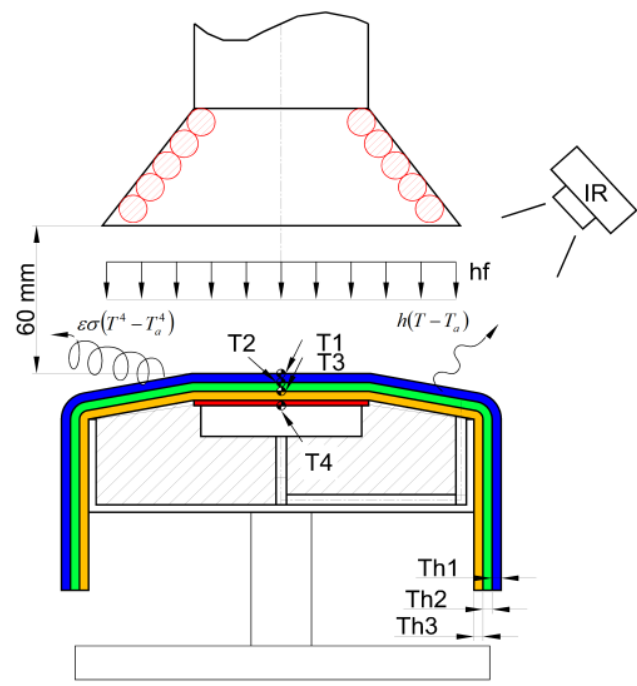

a) Configuração do dos ensaios no IPB.

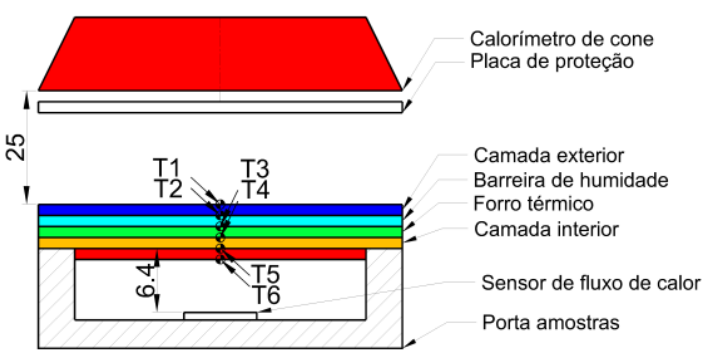

b) Configuração dos ensaios na Universidade de Tsinghua - China (Fu, Weng, and Yuan 2014).

Figura 1: Equipamento experimental para teste de conjunto de tecidos para

EPIs submetidos a radiação térmica num calorímetro de cone.

\section{TESTES EXPERIMENTAIS}

O desempenho térmico dos conjuntos dos tecidos montados é apresentado nas duas secções seguintes. A primeira secção apresenta os resultados experimentais obtidos na TU, China, enquanto a segunda secção apresenta os resultados experimentais obtidos no IPB, Portugal.

\subsection{Testes desenvolvidos em Tsinghua University (China)}

O objetivo deste estudo foi investigar os efeitos da radiação térmica de baixo nível no desempenho da proteção térmica de roupas de proteção secas e húmidas. Somente os resultados dos testes a seco são apresentados neste artigo, considerando dois conjuntos de tecidos diferentes, identificados como conjunto 01 e 02, ver Tabela 1. 
Tabela 1: Tecidos usados nos EPI's de bombeiros (multicamadas) (FU, Weng. and YUAN 2014).

\begin{tabular}{|c|c|c|c|c|c|c|c|}
\hline \multirow[b]{2}{*}{$\begin{array}{l}\text { CAMA } \\
\text { DA } \\
\text { ID. }\end{array}$} & \multirow[b]{2}{*}{$\begin{array}{c}\text { TIPO } \\
\text { TECIDO }\end{array}$} & \multicolumn{3}{|c|}{ CONJUNTO 01} & \multicolumn{3}{|c|}{ CONJUNTO 02} \\
\hline & & $\begin{array}{c}\text { ESPES } \\
\text { SURA } \\
{[\mathrm{mm}]}\end{array}$ & $\begin{array}{c}\text { NOME } \\
\text { COMERC } \\
\text { IAL }\end{array}$ & $\begin{array}{c}\text { DENSID } \\
\text { ADE } \\
{\left[\mathrm{g} / \mathrm{m}^{2}\right]}\end{array}$ & $\begin{array}{c}\text { ESPESS } \\
\text { URA } \\
\text { [mm] }\end{array}$ & $\begin{array}{c}\text { NOME } \\
\text { COMERC } \\
\text { IAL }\end{array}$ & $\begin{array}{c}\text { DENSID } \\
\text { ADE } \\
{\left[\mathrm{g} / \mathrm{m}^{2}\right]}\end{array}$ \\
\hline 1 & $\begin{array}{l}\text { Camada } \\
\text { exterior }\end{array}$ & 0.44 & Nomex & 200 & 0.44 & Nomex & 200 \\
\hline 2 & $\begin{array}{l}\text { Barreira de } \\
\text { humidade }\end{array}$ & 0.62 & $\begin{array}{l}\text { Neo- } \\
\text { Guard } \\
\text { (PTFE) }\end{array}$ & 105 & 0.12 & $\begin{array}{l}\text { Breath- } \\
\text { Tex Plus } \\
\text { (Goretex) }\end{array}$ & 119 \\
\hline 3 & $\begin{array}{l}\text { Forro } \\
\text { térmico }\end{array}$ & 2.60 & Aralite & 150 & 2.60 & Aralite & 150 \\
\hline 4 & $\begin{array}{l}\text { Camada } \\
\text { interior }\end{array}$ & 0.31 & $\begin{array}{l}\text { "Plain } \\
\text { weave" }\end{array}$ & 120 & 0.31 & $\begin{array}{l}\text { "Plain } \\
\text { weave" }\end{array}$ & 120 \\
\hline
\end{tabular}

As amostras foram precondicionadas numa câmara de temperatura e humidade ( $20 \pm 2 \stackrel{\circ}{\circ}$ e $65 \pm 3 \% \mathrm{HR}$ ) por 24 horas e, de seguida, selados em sacos plásticos por mais 24 horas antes do ensaio. Foram colocados Termopares do tipo K, com diâmetro de $0.4 \mathrm{~mm}$, na superfície superior de cada camada, para medir a distribuição de temperatura através de cada conjunto.

Cada amostra de teste foi colocada por baixo do calorímetro de cone, a uma distância de 25 [mm] e submetida a um nível de densidade de fluxo de calor incidente $Q_{0}$ de $1,2,3,5,7$ e $10\left[\mathrm{~kW} / \mathrm{m}^{2}\right]$. A curva do histórico da temperatura é apresentada apenas para $5\left[\mathrm{~kW} / \mathrm{m}^{2}\right]$, ver Figura 2. A principal diferença entre o comportamento do conjunto 01 e do conjunto 02 está relacionada com a temperatura T3 (temperatura medida na superfície superior da camada 2).

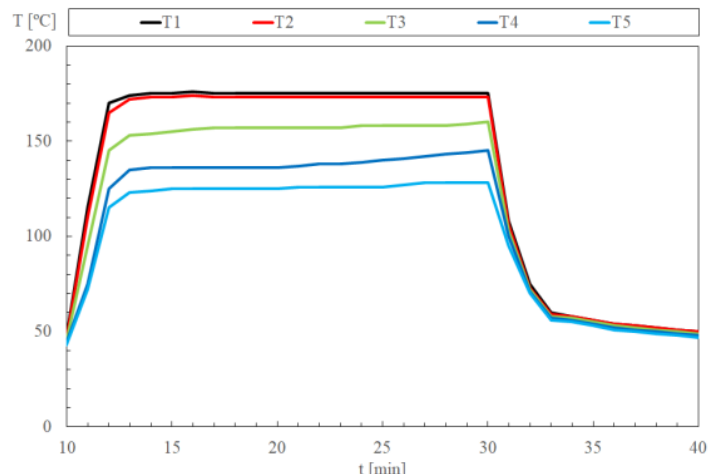

a) Conjunto 01 submetido a $5 \mathrm{~kW} / \mathrm{m}^{2}$.

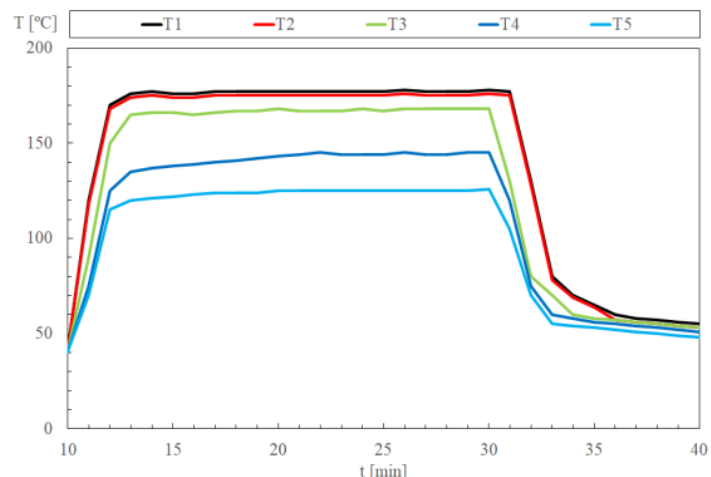

b) Conjunto 02 submetido a $5 \mathrm{~kW} / \mathrm{m}^{2}$. Figura 2: Teste de radiação no calorímetro de cone para análise de roupas de bombeiros a $5\left[\mathrm{KW} / \mathrm{m}^{2}\right]$. 
De acordo com os resultados experimentais, o fluxo de calor transmitido através conjunto para o lado não exposto é de $0.75\left[\mathrm{KW} / \mathrm{m}^{2}\right]$ e $0.675\left[\mathrm{~kW} / \mathrm{m}^{2}\right]$. respetivamente para os conjuntos 01 e 02 . O fator de transmissão de calor TF (Q0) para esse fluxo de calor é $15 \%$ e $13.5 \%$, respetivamente. Fu et al. (Fu, Weng, and Yuan 2014) apresentaram uma aproximação para o fator de transmissão de calor, com base na análise do ajuste de valores entre o fluxo de calor incidente e o fluxo de calor transmitido, ver Eq. 3 e 4 para montagem 01 e 02, respetivamente. Estas fórmulas proporcionam baixos valores para os fatores de transmissão de calor (TF), em comparação com os resultados experimentais realizados no IPB.

$$
\begin{aligned}
& Q_{c}=0.151 \cdot Q_{0}-0.016 \\
& Q_{c}=0.142 \cdot Q_{0}-0.018
\end{aligned}
$$

\subsection{Testes desenvolvidos no Instituto Politécnico de Bragança (Portugal)}

O objetivo deste estudo foi investigar os efeitos da radiação térmica de baixa intensidade $\left(5\left[\mathrm{~kW} / \mathrm{m}^{2}\right]\right)$ e de média intensidade $\left(20\left[\mathrm{~kW} / \mathrm{m}^{2}\right]\right)$ no desempenho térmico para o conjunto de tecidos de proteção utilizados em EPIs. Os ensaios foram realizados a seco, utilizando um conjunto de tecidos com 3 camadas. Foram ensaiados diferentes tipos de tecidos para cada uma das 3 camadas (ver Tabela 2), em particular 6 camadas exteriores diferentes, 4 barreiras térmicas e 3 tipos de camadas interiores. 
Tabela 2: Tipos de tecidos utilizados nos conjuntos.

\begin{tabular}{|c|c|c|c|c|}
\hline $\begin{array}{l}\text { CAMADA } \\
\text { ID. }\end{array}$ & $\begin{array}{l}\text { TIPO DE } \\
\text { TECIDO }\end{array}$ & $\begin{array}{c}\text { ESPESSURA } \\
\text { Th [mm] }\end{array}$ & $\begin{array}{l}\text { NOME COMERCIAL } \\
\text { COMPOSIÇÃO }\end{array}$ & $\begin{array}{c}\text { DENSIDADE } \\
\left(\mathrm{g} / \mathrm{m}^{2}\right)\end{array}$ \\
\hline TB1 & $\begin{array}{l}\text { Camada } \\
\text { exterior }\end{array}$ & 0.46 & 99\% Kermel / 1\% Carbon & 240 \\
\hline TB2 & $\begin{array}{l}\text { Camada } \\
\text { exterior }\end{array}$ & 0.47 & $\begin{array}{c}88 \% \text { Kermel / } 10 \% \text { Para-aramid / 2\% } \\
\text { Carbon }\end{array}$ & 250 \\
\hline TB3 & $\begin{array}{l}\text { Camada } \\
\text { exterior }\end{array}$ & 0.45 & $\begin{array}{c}82 \% \text { Kermel / } 16 \% \text { Para-aramid / 2\% } \\
\text { Carbon }\end{array}$ & 210 \\
\hline TB4 & $\begin{array}{l}\text { Camada } \\
\text { exterior }\end{array}$ & 0.38 & $\begin{array}{c}50 \% \text { Aramid (Kermel) / 25\% } \\
\text { Modacrylic / 24\% Lyocell / 1\% } \\
\text { Polyamide (Antistatic) }\end{array}$ & 230 \\
\hline TB5 & $\begin{array}{l}\text { Camada } \\
\text { exterior }\end{array}$ & 0.39 & $\begin{array}{c}64 \% \text { Kermel / 35\% Para-aramid / 1\% } \\
\text { Carbon }\end{array}$ & 200 \\
\hline TB6 & $\begin{array}{l}\text { Camada } \\
\text { exterior }\end{array}$ & 0.37 & $\begin{array}{c}60 \% \text { Aramid ( } 50 \% \text { Meta-aramid } \\
\text { (Kermel) }+10 \% \text { Para-aramid) } / 40 \% \\
\text { Viscose (LenzingFR) } \\
\end{array}$ & 230 \\
\hline BT1 & $\begin{array}{l}\text { Forro } \\
\text { térmico }\end{array}$ & 0.60 & $\begin{array}{l}\text { 45\% Kermel / 30\% PTFE / 25\% } \\
\text { Para-aramid }\end{array}$ & 140 \\
\hline BT2 & $\begin{array}{l}\text { Forro } \\
\text { térmico }\end{array}$ & 0.79 & $\begin{array}{c}37.5 \% \text { Aramid / } 25 \% \text { PTFE/PU FR / } \\
37.5 \% \text { Polyacrylate }\end{array}$ & 200 \\
\hline BT3 & $\begin{array}{l}\text { Forro } \\
\text { térmico }\end{array}$ & 0.50 & $\begin{array}{c}\text { 69\% Meta-Para-Aramid / 31\% } \\
\text { PTFE/PU }\end{array}$ & 105 \\
\hline BT4 & $\begin{array}{l}\text { Forro } \\
\text { térmico }\end{array}$ & 0.53 & $\begin{array}{c}\text { 47\% Meta -Aramid / 30\% PTFE/PU } \\
\text { FR / 23\% Para-Aramid }\end{array}$ & 140 \\
\hline $\mathrm{F} 1$ & $\begin{array}{l}\text { Camada } \\
\text { interior }\end{array}$ & 0.20 & 50\% Kermel / 50\%Lenzig FR & 130 \\
\hline $\mathrm{F} 2$ & $\begin{array}{l}\text { Camada } \\
\text { interior }\end{array}$ & 0.18 & 50\% Kermel / 50\%Lenzig FR & 110 \\
\hline F3 & $\begin{array}{l}\text { Camada } \\
\text { interior }\end{array}$ & 0.18 & $99 \%$ Kermel / 1\%Carbon & 90 \\
\hline
\end{tabular}

Foram realizados 72 ensaios para um nível de radiação baixo ( 5 $\left.\left[\mathrm{kW} / \mathrm{m}^{2}\right]\right)$ e 12 ensaios para um nível de radiação médio $\left(20\left[\mathrm{~kW} / \mathrm{m}^{2}\right]\right)$. As amostras ou conjuntos foram ensaiados a partir das condições iniciais de temperatura e humidade ambiente. Os resultados estão apresentados na tabela 3 para todas as combinações testadas, em particular, são apresentados os tempos $t_{12}$ e $t_{24}$ que representam o tempo necessário para elevar a temperatura média inicial (T4) em $12\left[{ }^{\circ} \mathrm{C}\right]$ e $24\left[{ }^{\circ} \mathrm{C}\right]$, respetivamente. Estas medições diretas foram utilizadas para o cálculo da densidade de fluxo transmitida $Q_{c}$ e do fator de transmissão de calor $\operatorname{TF}\left(Q_{0}\right)$, utilizando as Eqs. 1 e 2. 
Tabela 3: Combinações de tecidos usados em conjuntos de EPIs.

\begin{tabular}{|c|c|c|c|c|c|c|c|c|c|}
\hline TESTE & CONJ. & $\begin{array}{c}\mathbf{Q 0} \\
{\left[\mathrm{kW} / \mathrm{m}^{2}\right]}\end{array}$ & $\begin{array}{c}\text { CAMADA } \\
\text { EXTERIOR }\end{array}$ & $\begin{array}{c}\text { FORRO } \\
\text { TÉRMICO }\end{array}$ & $\begin{array}{l}\text { CAMADA } \\
\text { INTERIOR }\end{array}$ & $\begin{array}{l}t_{12} \\
{[s]}\end{array}$ & $\begin{array}{l}t_{24} \\
{[s]}\end{array}$ & $\begin{array}{c}\mathbf{Q}_{\mathrm{c}} \\
{\left[\mathrm{kW} / \mathrm{m}^{2}\right]}\end{array}$ & $\begin{array}{l}\text { TF } \\
\left(Q_{0}\right)\end{array}$ \\
\hline 1 & 1 & 5 & TB1 & BT1 & $F 1$ & 39.96 & 81.99 & 1.58 & $32 \%$ \\
\hline 2 & 2 & 5 & TB2 & BT1 & $\mathrm{F} 1$ & 63.38 & 101.08 & 1.76 & $35 \%$ \\
\hline 3 & 3 & 5 & TB3 & BT1 & F1 & 64.21 & 101.83 & 1.76 & $35 \%$ \\
\hline 4 & 4 & 5 & TB4 & BT1 & F1 & 41.30 & 77.02 & 1.86 & $37 \%$ \\
\hline 5 & 1 & 20 & TB1 & BT1 & $\mathrm{F} 1$ & 15.90 & 28.37 & 5.32 & $27 \%$ \\
\hline 6 & 2 & 20 & TB2 & BT1 & F1 & 16.00 & 25.37 & 7.08 & $35 \%$ \\
\hline 7 & 3 & 20 & TB3 & BT1 & $\mathrm{F} 1$ & 29.31 & 38.48 & 7.23 & $36 \%$ \\
\hline 8 & 4 & 20 & TB4 & BT1 & F1 & 13.66 & 21.10 & 8.91 & $45 \%$ \\
\hline 9 & 5 & 5 & TB5 & BT1 & F1 & 54.96 & 95.78 & 1.62 & $32 \%$ \\
\hline 10 & 6 & 5 & TB6 & BT1 & F1 & 48.96 & 86.18 & 1.78 & $36 \%$ \\
\hline 11 & 7 & 5 & TB1 & BT2 & F1 & 59.03 & 99.41 & 1.64 & $33 \%$ \\
\hline 12 & 8 & 5 & TB2 & BT2 & $\mathrm{F} 1$ & 54.54 & 90.98 & 1.82 & $36 \%$ \\
\hline 13 & 9 & 5 & TB3 & BT2 & $\mathrm{F} 1$ & 63.94 & 107.66 & 1.52 & $30 \%$ \\
\hline 14 & 10 & 5 & TB4 & BT2 & F1 & - & - & - & - \\
\hline 15 & 11 & 5 & TB5 & BT2 & F1 & 69.45 & 123.80 & 1.22 & $24 \%$ \\
\hline 16 & 12 & 5 & TB6 & BT2 & F1 & 73.92 & 132.00 & 1.14 & $23 \%$ \\
\hline 17 & 13 & 5 & TB1 & BT3 & $\mathrm{F} 1$ & 48.70 & 84.30 & 1.86 & $37 \%$ \\
\hline 18 & 14 & 5 & TB2 & BT3 & $\mathrm{F} 1$ & 41.41 & 70.72 & 2.26 & $45 \%$ \\
\hline 19 & 15 & 5 & TB3 & BT3 & $\mathrm{F} 1$ & 41.70 & 73.76 & 2.07 & $41 \%$ \\
\hline 20 & 16 & 5 & TB4 & BT3 & $\mathrm{F} 1$ & 39.40 & 68.51 & 2.28 & $46 \%$ \\
\hline 21 & 17 & 5 & TB5 & BT3 & F1 & 43.81 & 75.41 & 2.10 & $42 \%$ \\
\hline 22 & 18 & 5 & TB6 & BT3 & F1 & 46.36 & 79.75 & 1.99 & $40 \%$ \\
\hline 23 & 19 & 5 & TB1 & BT4 & F1 & 47.49 & 81.03 & 1.98 & $40 \%$ \\
\hline 24 & 20 & 5 & TB2 & BT4 & F1 & 47.78 & 79.27 & 2.11 & $42 \%$ \\
\hline 25 & 21 & 5 & TB3 & BT4 & F1 & 47.38 & 82.18 & 1.91 & $38 \%$ \\
\hline 26 & 22 & 5 & TB4 & BT4 & F1 & 53.37 & 89.66 & 1.83 & $37 \%$ \\
\hline 27 & 23 & 5 & TB5 & BT4 & F1 & 44.13 & 74.09 & 2.21 & $44 \%$ \\
\hline 28 & 24 & 5 & TB6 & BT4 & F1 & 47.34 & 79.51 & 2.06 & $41 \%$ \\
\hline 29 & 25 & 5 & TB1 & BT1 & F2 & 54.38 & 89.32 & 1.90 & $38 \%$ \\
\hline 30 & 26 & 5 & TB2 & BT1 & F2 & 46.44 & 80.70 & 1.94 & $39 \%$ \\
\hline 31 & 27 & 5 & TB3 & BT1 & F2 & 47.77 & 80.24 & 2.04 & $41 \%$ \\
\hline 32 & 28 & 5 & TB4 & BT1 & F2 & 48.27 & 80.47 & 2.06 & $41 \%$ \\
\hline 33 & 29 & 5 & TB5 & BT1 & F2 & 48.05 & 82.45 & 1.93 & $39 \%$ \\
\hline 34 & 30 & 5 & TB6 & BT1 & F2 & 45.66 & 81.37 & 1.86 & $37 \%$ \\
\hline 35 & 31 & 5 & TB1 & BT2 & F2 & 52.54 & 87.16 & 1.92 & $38 \%$ \\
\hline 36 & 32 & 5 & TB2 & BT2 & F2 & 55.80 & 92.51 & 1.81 & $36 \%$ \\
\hline 37 & 33 & 5 & TB3 & BT2 & F2 & 58.51 & 99.02 & 1.64 & $33 \%$ \\
\hline 38 & 34 & 5 & TB4 & BT2 & $\mathrm{F} 2$ & 50.34 & 86.21 & 1.85 & $37 \%$ \\
\hline 39 & 35 & 5 & TB5 & BT2 & F2 & 50.92 & 86.48 & 1.87 & $37 \%$ \\
\hline 40 & 36 & 5 & TB6 & BT2 & F2 & 53.24 & 78.25 & 2.65 & $53 \%$ \\
\hline 41 & 37 & 5 & TB1 & BT3 & F2 & 42.34 & 71.93 & 2.24 & $45 \%$ \\
\hline 42 & 38 & 5 & TB2 & BT3 & F2 & 46.03 & 76.52 & 2.17 & $43 \%$ \\
\hline 43 & 39 & 5 & TB3 & BT3 & F2 & 54.54 & 87.08 & 2.04 & $41 \%$ \\
\hline 44 & 40 & 5 & TB4 & BT3 & F2 & 44.09 & 75.49 & 2.11 & $42 \%$ \\
\hline 45 & 41 & 5 & TB5 & BT3 & F2 & 45.33 & 74.84 & 2.25 & $45 \%$ \\
\hline 46 & 42 & 5 & TB6 & BT3 & F2 & 44.72 & 74.61 & 2.22 & $44 \%$ \\
\hline 47 & 43 & 5 & TB1 & BT4 & F2 & 46.88 & 77.90 & 2.14 & $43 \%$ \\
\hline 48 & 44 & 5 & TB2 & BT4 & F2 & 50.33 & 83.96 & 1.97 & $39 \%$ \\
\hline
\end{tabular}




\begin{tabular}{|c|c|c|c|c|c|c|c|c|c|}
\hline 49 & 45 & 5 & TB3 & BT4 & $\mathrm{F} 2$ & 55.21 & 93.04 & 1.75 & $35 \%$ \\
\hline 50 & 46 & 5 & TB4 & BT4 & F2 & 53.08 & 87.69 & 1.92 & $38 \%$ \\
\hline 51 & 47 & 5 & TB5 & BT4 & $\mathrm{F} 2$ & 41.92 & 72.83 & 2.15 & $43 \%$ \\
\hline 52 & 48 & 5 & TB6 & BT4 & $\mathrm{F} 2$ & 45.43 & 76.39 & 2.14 & $43 \%$ \\
\hline 53 & 49 & 5 & TB1 & BT1 & F3 & 47.09 & 79.31 & 2.06 & $41 \%$ \\
\hline 54 & 50 & 5 & TB2 & BT1 & F3 & 45.13 & 76.59 & 2.11 & $42 \%$ \\
\hline 55 & 51 & 5 & TB3 & BT1 & F3 & 38.68 & 66.81 & 2.36 & $47 \%$ \\
\hline 56 & 52 & 5 & TB4 & BT1 & F3 & 46.04 & 79.84 & 1.96 & $39 \%$ \\
\hline 57 & 53 & 5 & TB5 & BT1 & F3 & 41.30 & 70.67 & 2.26 & $45 \%$ \\
\hline 58 & 54 & 5 & TB6 & BT1 & F3 & 42.12 & 74.27 & 2.06 & $41 \%$ \\
\hline 59 & 55 & 5 & TB1 & BT2 & F3 & 46.76 & 78.64 & 2.08 & $42 \%$ \\
\hline 60 & 56 & 5 & TB2 & BT2 & F3 & 54.47 & 91.17 & 1.81 & $36 \%$ \\
\hline 61 & 57 & 5 & TB3 & BT2 & F3 & 55.32 & 90.29 & 1.90 & $38 \%$ \\
\hline 62 & 58 & 5 & TB4 & BT2 & F3 & 50.37 & 85.81 & 1.87 & $37 \%$ \\
\hline 63 & 59 & 5 & TB5 & BT2 & F3 & 50.49 & 84.84 & 1.93 & $39 \%$ \\
\hline 64 & 60 & 5 & TB6 & BT2 & F3 & 53.74 & 91.49 & 1.76 & $35 \%$ \\
\hline 65 & 61 & 5 & TB1 & BT3 & F3 & 43.72 & 74.11 & 2.18 & $44 \%$ \\
\hline 66 & 62 & 5 & TB2 & BT3 & F3 & 39.32 & 66.20 & 2.47 & $49 \%$ \\
\hline 67 & 63 & 5 & TB3 & BT3 & F3 & 47.31 & 81.12 & 1.96 & $39 \%$ \\
\hline 68 & 64 & 5 & TB4 & BT3 & F3 & 45.11 & 76.26 & 2.13 & $43 \%$ \\
\hline 69 & 65 & 5 & TB5 & BT3 & F3 & 42.78 & 74.87 & 2.07 & $41 \%$ \\
\hline 70 & 66 & 5 & TB6 & BT3 & F3 & 39.36 & 68.81 & 2.25 & $45 \%$ \\
\hline 71 & 67 & 5 & TB1 & BT4 & F3 & 45.11 & 75.92 & 2.15 & $43 \%$ \\
\hline 72 & 68 & 5 & TB2 & BT4 & F3 & 43.22 & 73.72 & 2.17 & $43 \%$ \\
\hline 73 & 69 & 5 & TB3 & BT4 & F3 & 48.79 & 146.03 & 0.68 & $14 \%$ \\
\hline 74 & 70 & 5 & TB4 & BT4 & F3 & 47.27 & 79.23 & 2.08 & $42 \%$ \\
\hline 75 & 71 & 5 & TB5 & BT4 & F3 & 44.22 & 76.55 & 2.05 & $41 \%$ \\
\hline 76 & 72 & 5 & TB6 & BT4 & F3 & 44.70 & 76.62 & 2.08 & $42 \%$ \\
\hline 77 & 5 & 20 & TB5 & BT1 & $\mathrm{F} 1$ & 15.30 & 24.74 & 7.02 & $35 \%$ \\
\hline 78 & 6 & 20 & TB6 & BT1 & $\mathrm{F} 1$ & 14.23 & 21.72 & 8.85 & $44 \%$ \\
\hline 79 & 7 & 20 & TB1 & BT2 & $\mathrm{F} 1$ & 18.92 & 29.23 & 6.44 & $32 \%$ \\
\hline 80 & 8 & 20 & TB2 & BT2 & $\mathrm{F} 1$ & 19.94 & 29.55 & 6.90 & $35 \%$ \\
\hline 81 & 9 & 20 & TB3 & BT2 & $\mathrm{F} 1$ & 21.73 & 34.23 & 5.30 & $27 \%$ \\
\hline 82 & 10 & 20 & TB4 & BT2 & $\mathrm{F} 1$ & 20.54 & 31.06 & 6.31 & $32 \%$ \\
\hline 83 & 11 & 20 & TB5 & BT2 & $\mathrm{F} 1$ & 23.08 & 35.91 & 5.17 & $26 \%$ \\
\hline 84 & 12 & 20 & TB6 & BT2 & $\mathrm{F} 1$ & 22.33 & 34.83 & 5.31 & $27 \%$ \\
\hline
\end{tabular}

A seleção da melhor combinação foi verificada através da comparação do comportamento dos índices de transmissibilidade, fixando um tipo de camada e fazendo variar os restantes dois tipos. Na tabela 4 é apresentada a melhor combinação para a camada exterior, quando submetidas as amostras a uma densidade de fluxo de calor incidente de $5\left[\mathrm{~kW} / \mathrm{m}^{2}\right]$, fixando as camadas TBi ( $i=1 . .6)$ e fazendo variar os restantes tipos de camadas. A camada TB3 apresenta menor valor de densidade de fluxo de calor transmitido, ao qual corresponde o menor fator de transmissão de calor. 
Tabela 4: Resultados com TBi em conjuntos de EPIs, submetidos a $5\left[\mathrm{~kW} / \mathrm{m}^{2}\right]$.

\begin{tabular}{|c|c|c|c|c|}
\hline $\begin{array}{c}\text { CAMADA } \\
\text { ID. }\end{array}$ & $\begin{array}{c}\text { ESPESSURA } \\
\text { Th } \mathbf{~} \mathbf{m m}]\end{array}$ & $\begin{array}{c}\text { DENSIDADE } \\
\left(\mathbf{g} / \mathbf{m}^{\mathbf{2}} \mathbf{)}\right.\end{array}$ & $\begin{array}{c}\mathbf{Q c} \\
{\left[\mathbf{k w} / \mathbf{m}^{2}\right]}\end{array}$ & $\begin{array}{c}\text { TF } \\
{[\%]}\end{array}$ \\
\hline TB1 & 0.46 & 240 & $2.23 \pm 0.91$ & $39 \pm 5.0$ \\
\hline TB2 & 0.47 & 250 & $2.03 \pm 0.21$ & $41 \pm 4.0$ \\
\hline TB3 & 0.45 & 210 & $1.80 \pm 0.40$ & $36 \pm 8.0$ \\
\hline TB4 & 0.38 & 230 & $1.99 \pm 0.14$ & $40 \pm 3.0$ \\
\hline TB5 & 0.39 & 200 & $1.97 \pm 0.29$ & $39 \pm 6.0$ \\
\hline TB6 & 0.37 & 230 & $2.00 \pm 0.35$ & $40 \pm 7.0$ \\
\hline
\end{tabular}

Na tabela 5 é apresentada a melhor combinação para a barreira térmica, quando submetidas as amostras a uma densidade de fluxo de calor incidente de $5\left[\mathrm{~kW} / \mathrm{m}^{2}\right]$, fixando as camadas $\mathrm{BTi}(\mathrm{i}=1 . .4)$ e fazendo variar os restantes tipos de camadas. A camada BT2 apresenta menor valor de densidade de fluxo de calor transmitido, ao qual corresponde o menor fator de transmissão de calor.

Tabela 5: Resultados com BTi em conjuntos de EPIs, submetidos a $5\left[\mathrm{~kW} / \mathrm{m}^{2}\right]$.

\begin{tabular}{|c|c|c|c|c|}
\hline $\begin{array}{l}\text { CAMADA } \\
\text { ID. }\end{array}$ & $\begin{array}{l}\text { ESPESSURA } \\
\text { Th [mm] }\end{array}$ & $\begin{array}{c}\text { DENSIDADE } \\
\left(\mathrm{g} / \mathrm{m}^{2}\right)\end{array}$ & $\begin{array}{c}\mathbf{Q c} \\
{\left[\mathrm{kw} / \mathrm{m}^{2}\right]}\end{array}$ & $\begin{array}{c}\text { TF } \\
\text { [\%] }\end{array}$ \\
\hline BT1 & 0.6 & 140 & $1.94 \pm 0.20$ & $39 \pm 4.0$ \\
\hline BT2 & 0.79 & 200 & $1.79 \pm 0.32$ & $36 \pm 6.0$ \\
\hline BT3 & 0.5 & 105 & $2.15 \pm 0.14$ & $43 \pm 3.0$ \\
\hline BT4 & 0.53 & 140 & $1.97 \pm 0.33$ & $39 \pm 7.0$ \\
\hline
\end{tabular}

Na tabela 6 é apresentada a melhor combinação para a camada interior, quando submetidas as amostras a uma densidade de fluxo de calor incidente de $5\left[\mathrm{~kW} / \mathrm{m}^{2}\right]$, fixando as camadas $\mathrm{Fi}(\mathrm{i}=1 . .3)$ e fazendo variar os restantes tipos de camadas. A camada F1 apresenta menor valor de densidade de fluxo de calor transmitido, ao qual corresponde o menor fator de transmissão de calor. 
Tabela 6: Resultados com Fi em conjuntos de EPIs, submetidos a $5\left[\mathrm{~kW} / \mathrm{m}^{2}\right]$.

\begin{tabular}{|c|c|c|c|c|}
\hline $\begin{array}{c}\text { CAMADA } \\
\text { ID. }\end{array}$ & $\begin{array}{c}\text { ESPESSURA } \\
\text { Th }[\mathbf{m m}]\end{array}$ & $\begin{array}{c}\text { DENSIDADE } \\
\left(\mathbf{g} / \mathbf{m}^{\mathbf{2}}\right)\end{array}$ & $\begin{array}{c}\mathbf{Q c} \\
{\left[\mathbf{k w} / \mathbf{m}^{2}\right]}\end{array}$ & $\begin{array}{c}\text { TF } \\
{[\%]}\end{array}$ \\
\hline F1 & 0.2 & 130 & $1.84 \pm 0.29$ & $37 \pm 6.0$ \\
\hline F2 & 0.18 & 110 & $2.02 \pm 0.21$ & $40 \pm 4.0$ \\
\hline F3 & 0.18 & 90 & $2.02 \pm 0.32$ & $40 \pm 6.0$ \\
\hline
\end{tabular}

Em função dos resultados obtidos, pode concluir-se que o melhor conjunto de tecidos resulta da combinação TB3/BT2/F1, ver Figura 3, com imagens antes e depois do ensaio. $O$ conjunto 9 não apresenta degradação evidente das fibras que constituem a amostra, mantendo-se a integridade aparente do conjunto.

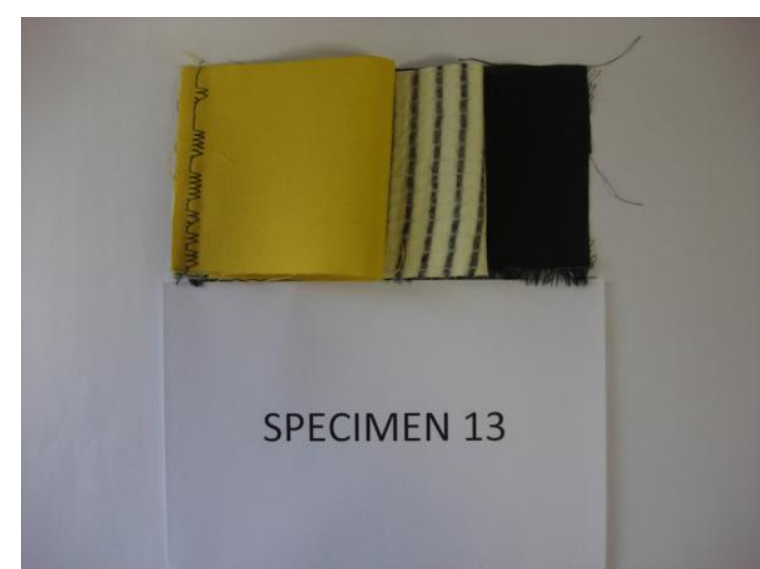

a) Antes do ensaio.

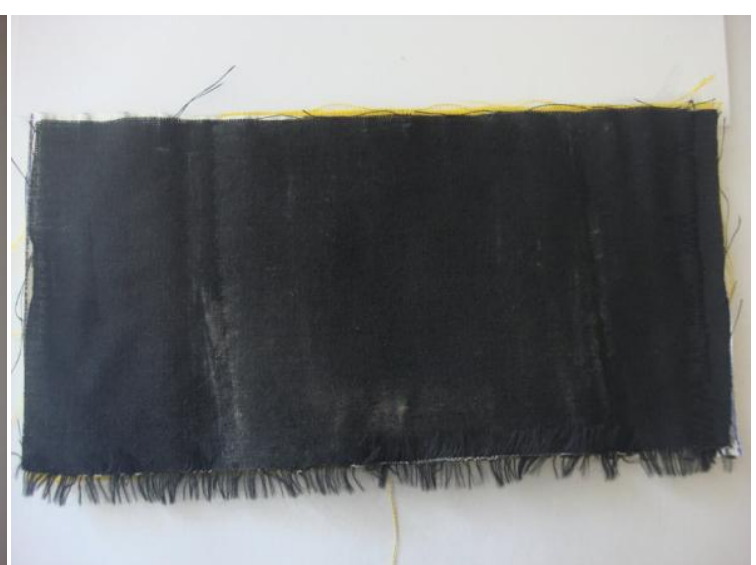

b) Após ensaio.

Figura 3: Conjunto 9 para combinação de tecidos TB3/BT2/F1.

No Figura 4 é apresentado o gráfico da evolução da temperatura para 0 ensaio do conjunto 9 , quando submetido a uma densidade de fluxo de 5 $\left[\mathrm{kW} / \mathrm{m}^{2}\right]$, e o campo de temperatura da imagem obtida por câmara termográfica de infravermelhos, após 5 [min] de exposição ao fluxo de calor radiante. A escala de temperatura da câmara termográfica foi fixada entre $15\left[{ }^{\circ} \mathrm{C}\right]$ e $350\left[{ }^{\circ} \mathrm{C}\right]$. 


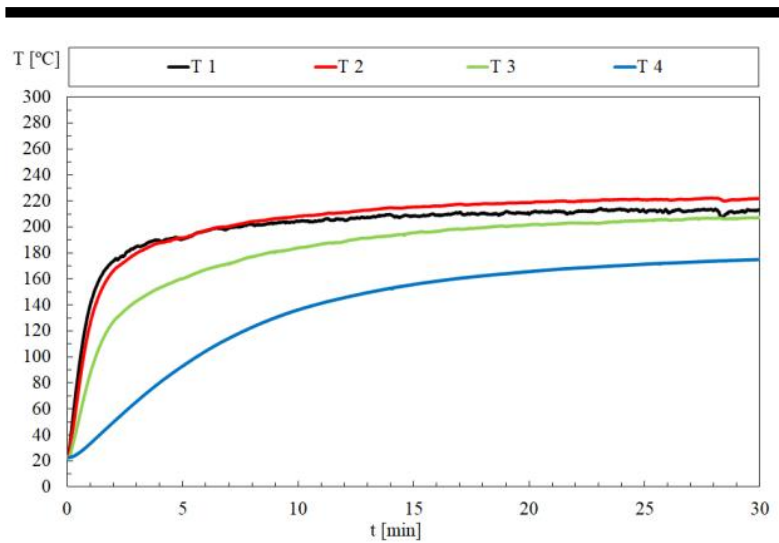

a) Gráfico de Temperatura

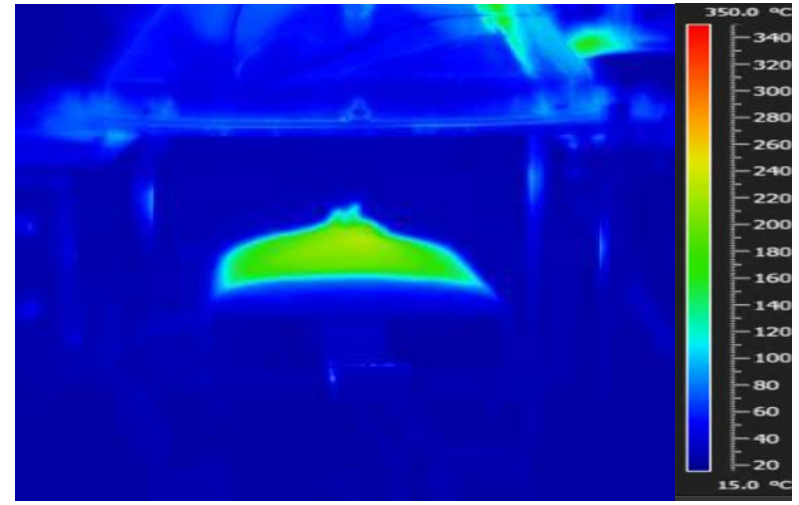

b) Imagem termográfica ao fim de 5 $\min$

Figura 4: Evolução de temperatura e imagem termográfica para combinação de tecidos TB3/BT2/F1 (Conjunto 9).

A evolução de temperatura estabiliza ao fim de 20 minutos, sendo a temperatura de cada camada quase constante e uniforme. O perfil de temperatura T4 apresenta uma taxa de variação inferior em comparação com outros conjuntos testados, conferindo a este conjunto o menor fator de transmissão de calor (30\%). De salientar que este fator é determinado na fase inicial do ensaio, entre os instantes 63 [s] e 107 [s].

Na tabela 7 é apresentada a melhor combinação para a camada exterior, quando as amostras são submetidas a uma densidade de fluxo de calor incidente de $20\left[\mathrm{~kW} / \mathrm{m}^{2}\right]$. Foi utilizado o mesmo procedimento para identificação da melhor camada. A camada TB1 apresenta menor valor de densidade de fluxo de calor transmitido, ao qual corresponde o menor fator de transmissão de calor, sendo que a camada TB3 apresenta o terceiro melhor resultado.

Tabela 7: Resultados com TBi em conjuntos de EPIs, submetidos a $20\left[\mathrm{~kW} / \mathrm{m}^{2}\right]$.

\begin{tabular}{|c|c|c|c|c|}
\hline $\begin{array}{c}\text { CAMADA } \\
\text { ID. }\end{array}$ & $\begin{array}{c}\text { ESPESSURA } \\
\text { Th }[\mathbf{m m}]\end{array}$ & $\begin{array}{c}\text { DENSIDADE } \\
\left(\mathbf{g} / \mathbf{m}^{\mathbf{2}}\right)\end{array}$ & $\begin{array}{c}\mathbf{Q C} \\
{\left[\mathbf{k w} / \mathbf{m}^{2}\right]}\end{array}$ & $\begin{array}{c}\text { TF } \\
{[\%]}\end{array}$ \\
\hline TB1 & 0.46 & 240 & $5.88 \pm 0.56$ & $29 \pm 3.0$ \\
\hline TB2 & 0.47 & 250 & $6.99 \pm 0.09$ & $35 \pm 0.4$ \\
\hline TB3 & 0.45 & 210 & $6.27 \pm 0.96$ & $31 \pm 5.0$ \\
\hline TB4 & 0.38 & 230 & $7.61 \pm 1.30$ & $38 \pm 7.0$ \\
\hline TB5 & 0.39 & 200 & $6.09 \pm 0.93$ & $30 \pm 5.0$ \\
\hline TB6 & 0.37 & 230 & $7.08 \pm 1.77$ & $35 \pm 9.0$ \\
\hline
\end{tabular}


Na tabela 8 é apresentada a melhor combinação para a barreira térmica, quando submetidas as amostras a uma densidade de fluxo de calor incidente de $20\left[\mathrm{~kW} / \mathrm{m}^{2}\right]$, fixando as camadas $\mathrm{BTi}(\mathrm{i}=1 . .2)$ e fazendo variar os restantes tipos de camadas. A camada BT2 apresenta menor valor de densidade de fluxo de calor transmitido, à semelhança do que acontece para o nível de radiação mais baixo.

Tabela 8: Resultados com BTi em conjuntos de EPIs, submetidos a $20\left[\mathrm{~kW} / \mathrm{m}^{2}\right]$.

\begin{tabular}{|c|c|c|c|c|}
\hline $\begin{array}{l}\text { CAMADA } \\
\text { ID. }\end{array}$ & $\begin{array}{l}\text { ESPESSURA } \\
\text { Th [mm] }\end{array}$ & $\begin{array}{c}\text { DENSIDADE } \\
\left(\mathrm{g} / \mathrm{m}^{2}\right)\end{array}$ & $\begin{array}{c}\mathbf{Q c} \\
{\left[\mathrm{kw} / \mathrm{m}^{2}\right]}\end{array}$ & $\begin{array}{c}\text { TF } \\
{[\%]}\end{array}$ \\
\hline BT1 & 0.6 & 140 & $7.04 \pm 1.23$ & $37 \pm 6.0$ \\
\hline BT2 & 0.79 & 200 & $5.90 \pm 0.67$ & $30 \pm 3.0$ \\
\hline
\end{tabular}

Na tabela 9 é apresentado o resultado da única camada interior combinada, quando as amostras são submetidas a uma densidade de fluxo de calor incidente de $20\left[\mathrm{~kW} / \mathrm{m}^{2}\right]$.

Tabela 9: Resultados com F1 em conjuntos de EPls, submetidos a $20\left[\mathrm{~kW} / \mathrm{m}^{2}\right]$.

\begin{tabular}{|c|c|c|c|c|}
\hline $\begin{array}{c}\text { CAMADA } \\
\text { ID. }\end{array}$ & $\begin{array}{c}\text { ESPESSURA } \\
\text { Th }[\mathbf{m m}]\end{array}$ & $\begin{array}{c}\text { DENSIDADE } \\
\left(\mathbf{g} / \mathbf{m}^{2}\right)\end{array}$ & $\begin{array}{c}\mathbf{Q C} \\
{\left[\mathbf{k w} / \mathbf{m}^{2}\right]}\end{array}$ & $\begin{array}{c}\text { TF } \\
{[\%]}\end{array}$ \\
\hline F1 & 0.2 & 130 & $6.65 \pm 1.24$ & $33 \pm 6.0$ \\
\hline
\end{tabular}

Em função dos resultados obtidos, pode concluir-se que o melhor conjunto de tecidos para as amostras submetidas a uma densidade de fluxo de calor de $20\left[\mathrm{~kW} / \mathrm{m}^{2}\right]$ é aquele que resulta da combinação TB1/BT2/F1, ver Figura 4, com imagens antes e depois do ensaio. O conjunto 7 apresenta degradação evidente das fibras que constituem a amostra, não sendo possível manter a integridade do conjunto. 


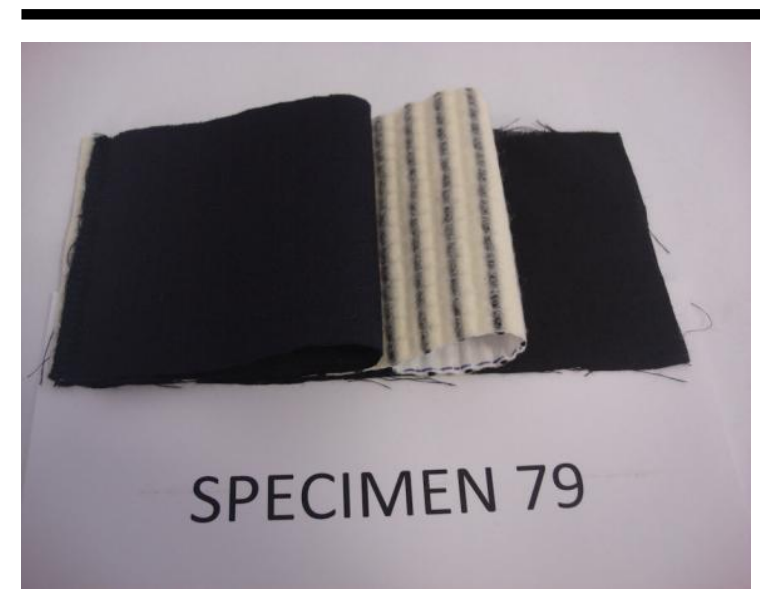

a) Antes do ensaio.

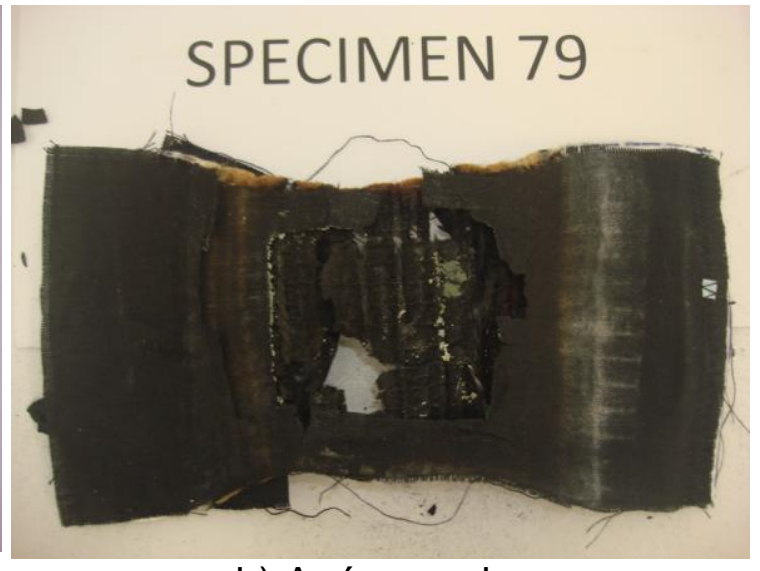

b) Após ensaio.

Figura 4: Conjunto 7 para combinação de tecidos TB1/BT2/F1.

No Figura 5 é apresentado o gráfico da evolução de temperaturas para o ensaio do conjunto 7, quando submetido a uma densidade de fluxo de 20 $\left[\mathrm{kW} / \mathrm{m}^{2}\right]$, e o campo de temperatura da imagem obtida por câmara termográfica de infravermelhos, após 5 [min] de exposição ao fluxo de calor radiante. A escala de temperatura da câmara termográfica também foi fixada entre 15 [ํㅡ] e $350\left[{ }^{\circ} \mathrm{C}\right]$.

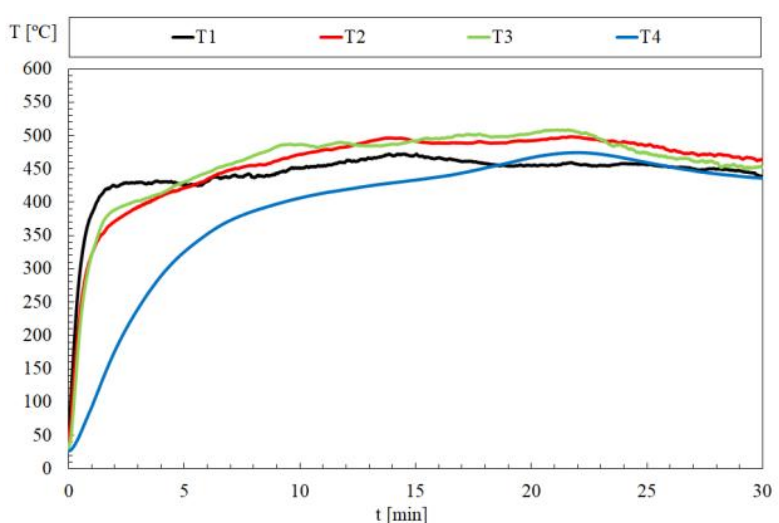

a) Gráfico de Temperatura.

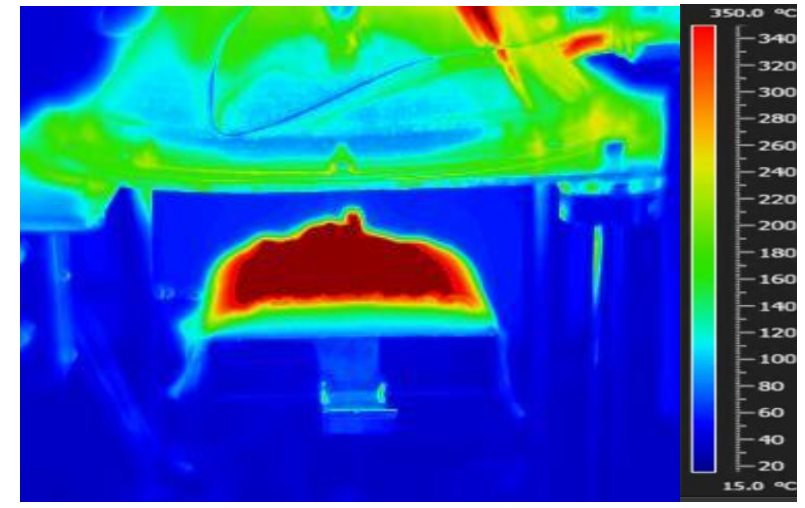

b) Imagem termográfica ao fim de 5 $\min$.

Figura 5: Evolução de temperatura e imagem termográfica para combinação de tecidos TB1/BT2/F1 (Conjunto 7).

Este conjunto sofreu degradação térmica durante o ensaio, motivo pelo qual se verifica que as temperaturas T2 e T3 acabam por ser superiores ao 
valor da medição de T1. Esta degradação torna-se visível após 5 [min] do início do ensaio. A Figura 5 b) apresenta o resultado do campo de temperatura na superfície exterior do tecido TB1, sendo que o valor da temperatura é superior a $400\left[^{\circ} \mathrm{C}\right]$, pelo que já se encontra fora do limite da escala termográfica.

O perfil de temperatura T4 apresenta uma taxa de variação inferior em comparação com outros conjuntos testados, conferindo a este conjunto o menor fator de transmissão de calor (32\%) para uma densidade de fluxo de calor de $20\left[\mathrm{~kW} / \mathrm{m}^{2}\right]$. De salientar que este fator é determinado na fase inicial do ensaio, entre os instantes 19 [s] e 29 [s]. O perfil de temperatura não atinge um regime estacionário, pelo contrário, verifica-se que ao fim de 20 [min] a temperatura T2, T3 e T4 diminui ligeiramente, provavelmente motivado pela degradação térmica das várias camadas do conjunto.

\section{SIMULAÇÃO E ANÁLISE PARAMÉTRICA DOS ENSAIOS TU CHINA}

Foi definido um modelo de elementos finitos 2D para o estudo do comportamento térmico dos conjuntos de tecidos, tendo em consideração a discretização da espessura de cada tecido. Os autores decidiram validar 0 modelo numérico usando os resultados experimentais desenvolvidos na Universidade de Tsinghua (China). O modelo assume $100 \mathrm{~mm}$ de largura e todas as espessuras definidas para os tecidos de cada montagem, ver Tabela 1. Este modelo usa o elemento PLANE55, que possui quatro nós, com um grau de liberdade por nó. Este elemento apresenta funções de interpolação linear e utiliza um esquema de integração total $(2 \times 2)$. Tendo em consideração a limitação deste elemento finito, foi criado um modelo adicional para receber 0 fluxo de calor incidente. O contacto entre os dois modelos é feito com um elemento finito diferente, COMBIN39, que utiliza funções de interpolação lineares e integração exata. ver Figura 6. 0 modelo também inclui a espessura da placa de cobre com $1.6[\mathrm{~mm}]$. O contacto entre todas as camadas e a placa de cobre é considerado perfeito. 


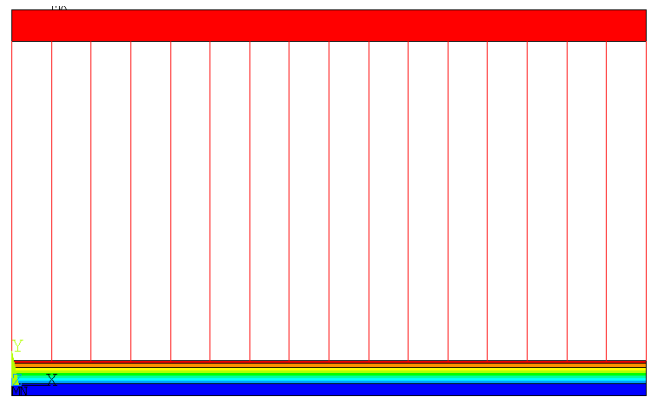

a) Malha de elementos finitos.

b) Conjunto 01 (4 camadas) submetida a 5 $\left[\mathrm{kW} / \mathrm{m}^{2}\right]$, após 5 minutos de exposição.

Figura 6: Malha de elementos finitos e campo de temperatura após 5 minutos de exposição ao calor.

$\mathrm{Na}$ superfície superior da camada exterior foi considerada a perda de calor por convecção e radiação $\left(\varepsilon_{f}=1\right)$. O coeficiente de convecção muda com 0 efeito da temperatura (Staggs and Whiteley 1999). A temperatura do ambiente é considerada igual a $20\left[^{\circ} \mathrm{C}\right]$. Uma condição térmica adiabática é aplicada na superfície inferior da placa de cobre e nas superfícies laterais das diferentes camadas. O tamanho do modelo difere do tamanho da amostra, uma vez que a área do modelo 2D é de 100 [mm] multiplicada pelo valor de cada espessura de tecido.

O modelo considera o comportamento não linear do material, com base nas propriedades térmicas dependentes da temperatura ( $\mathrm{J}$ Randall Lawson et al. 2005), obtidas por testes experimentais para baixos valores de temperatura $\left(20^{\circ} \mathrm{C}-73^{\circ} \mathrm{C}\right.$ ). $\mathrm{O}$ valor das propriedades foi extrapolado para níveis de temperatura superiores. As propriedades térmicas do cobre foram definidas de acordo com Hust et al. (Hust and Lankford 1984), ver Figura 7. O material do modelo adicional foi definido para permitir a passagem de fluxo de calor com baixíssima resistência térmica (elevada condutividade, $1000[\mathrm{~W} / \mathrm{mK}]$ e baixa capacidade térmica, $\left.1\left[\mathrm{~kg} / \mathrm{m}^{3}\right] \times 1[\mathrm{~J} / \mathrm{kgK}]\right)$. 


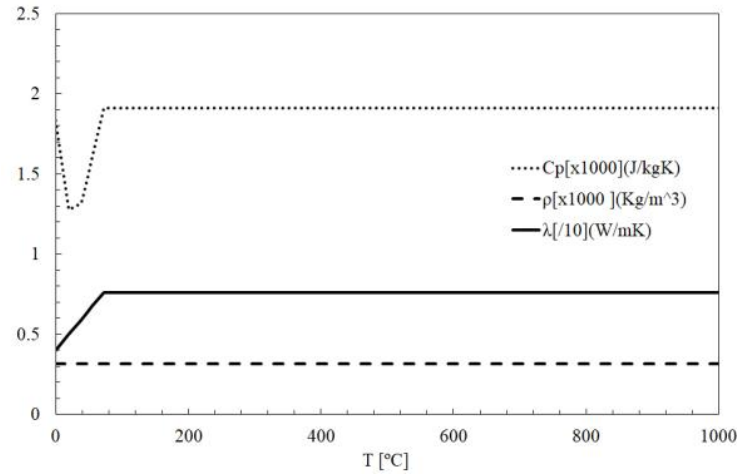

a) material da camada exterior.

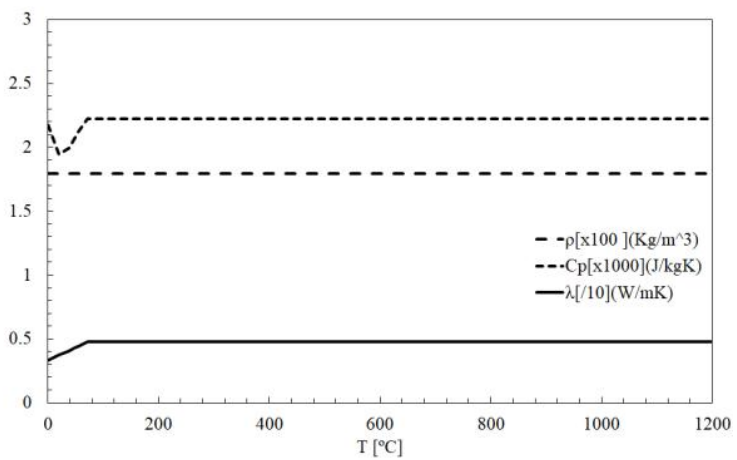

c) material da barreira de humidade (GORETEX).

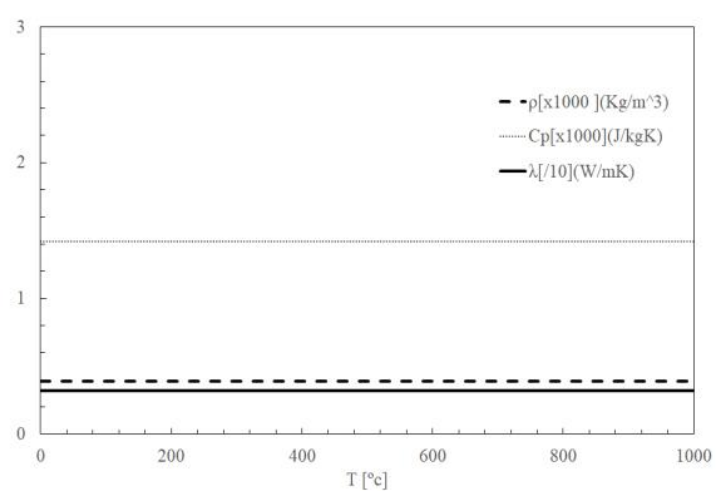

e) material da camada interior.

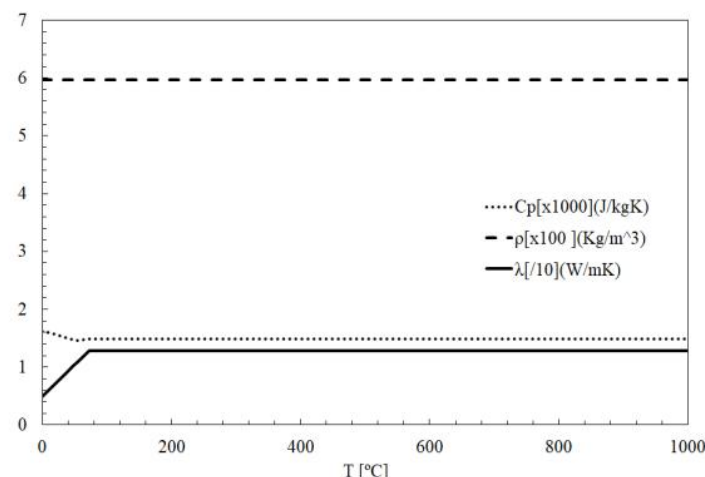

b) material da barreira de humidade (PTFE).

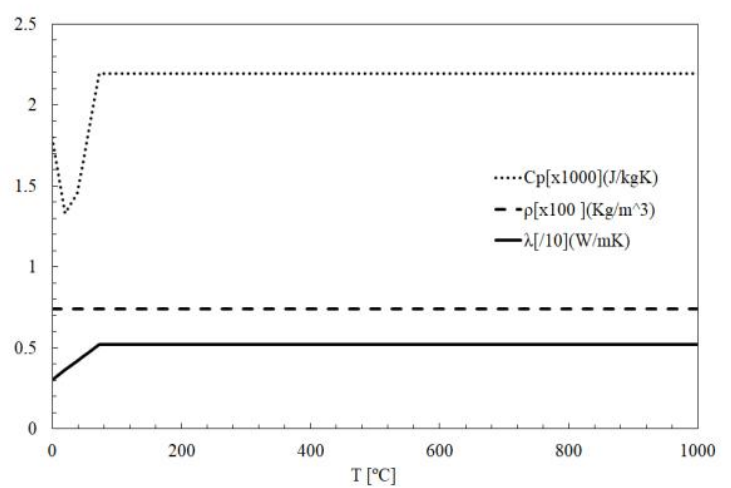

d) material do forro térmico.

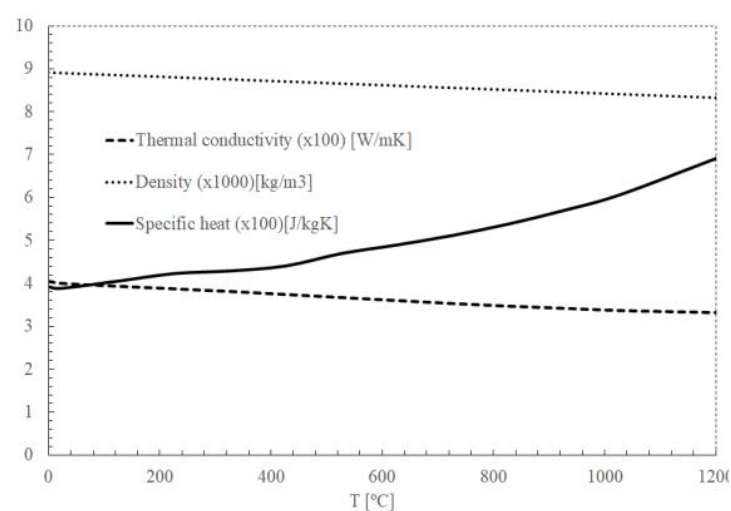

f) Cobre.

Figura 7: Propriedades térmicas dos materiais.

A solução é não linear e o incremento de tempo pode mudar de 60 [s] para 1 [s], dependendo do processo de convergência da solução. Os testes experimentais apresentam um estágio de aquecimento de 20 [min] e um estágio de arrefecimento de 10 [min]. 
Na Figura 8 é apresentada a evolução da temperatura de cada camada obtida através do modelo numérico. Estes resultados foram determinados para densidades de fluxo de calor de 5 e $10\left[\mathrm{~kW} / \mathrm{m}^{2}\right]$, sendo comparados com os resultados experimentais. $O$ modelo numérico é capaz de fazer uma boa previsão das temperaturas T1, T2 e T3, mas não consegue fazer uma aproximação dos valores de T4 e T5. Essa diferença pode ser explicada pelas condições fronteira utilizadas na superfície inferior da placa de cobre e ainda pela degradação térmica que poderá ter ocorrido nas camadas de tecido mais expostas, que naturalmente poderão favorecer o rápido aumento das temperaturas nestes pontos de medição.

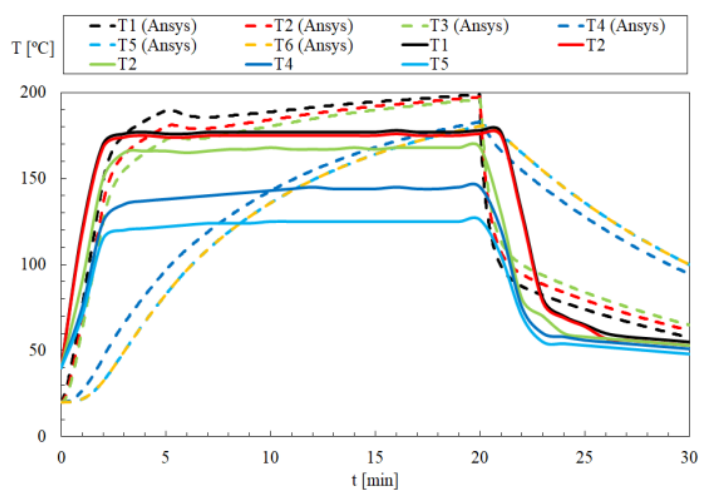

a) Conjunto 01 (4 camadas) submetida a $5\left[\mathrm{~kW} / \mathrm{m}^{2}\right]$.

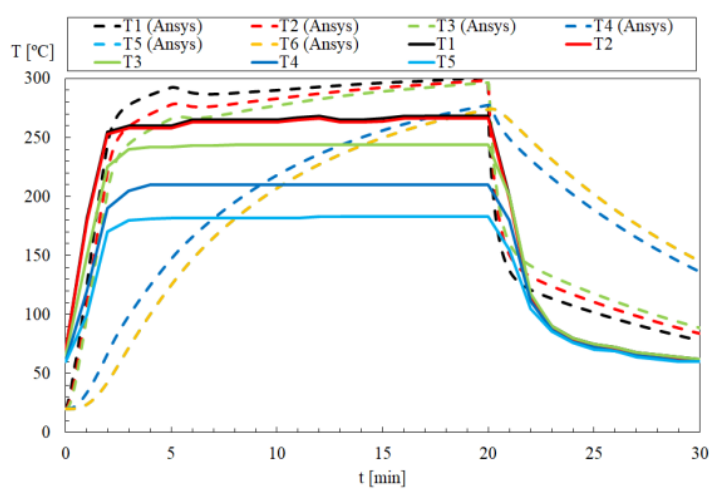

b) Conjunto 01 (4 camadas) submetida a $10\left[\mathrm{~kW} / \mathrm{m}^{2}\right]$.

Figura 8: Comparação de resultados dos ensaios em TU China, para o modelo com barreira de humidade PTFE.

Foi ainda efetuado um estudo paramétrico para avaliação do efeito da densidade de fluxo de calor incidente, fazendo variar o valor de $Q_{0}(1,2,3,5,7$, 10,20 e $\left.40\left[\mathrm{~kW} / \mathrm{m}^{2}\right]\right)$. Na Figura 9 está representada a relação não linear obtida entre o fluxo de calor incidente e o fluxo de calor transmitido. É também apresentada a relação entre o fluxo de calor incidente e o fator de transmissão de calor, sendo observado uma diminuição progressiva. Este comportamento parte do princípio que o modelo numérico mantém a integridade material durante o ensaio, situação que não foi verificada para os ensaios realizados no IPB, para os ensaios de valor médio de radiação. Estes resultados numéricos 
diferem dos resultados experimentais, pelo facto do perfil da temperatura T4 e T5 serem bastante diferentes.

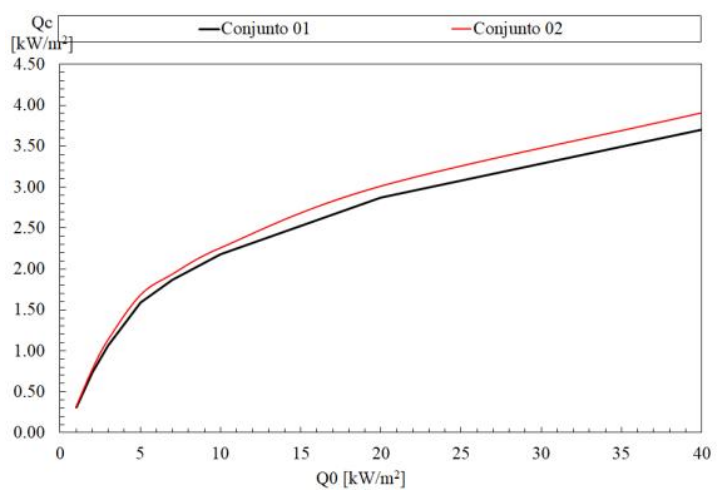

a) Fluxo de calor transmitido versus fluxo de calor incidente.

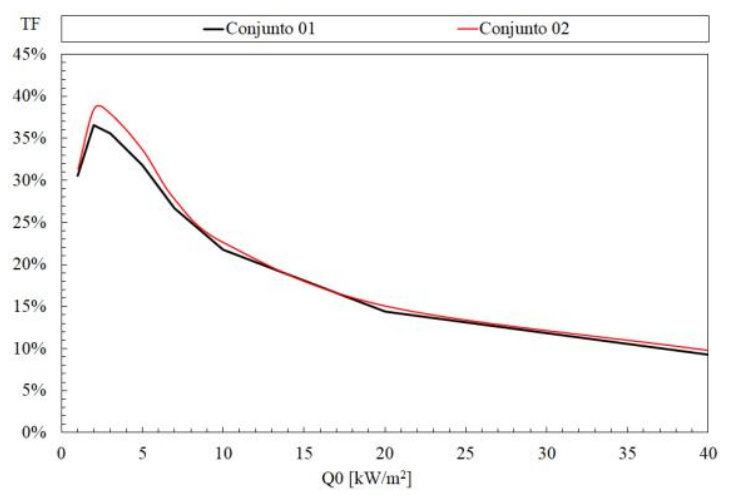

b) Fator de transmissão de calor.

Figura 9: resultados do estudo paramétrico.

\section{CONCLUSÕES}

Esta investigação apresenta os resultados de dois ensaios experimentais diferentes para avaliar o comportamento térmico de tecidos e conjuntos de tecidos quando expostos a uma fonte de calor radiante. Foram considerados pequenos e médios fluxos de calor radiante.

São apresentados resultados da temperatura entre cada camada. O conjunto dos tecidos ensaiados na TU China é composto por quatro camadas, enquanto que o conjunto de tecidos ensaiados no IPB é de apenas 3 camadas. O número de ensaios raziados em Portugal (84) foi muito superior ao número de ensaios realizados na TU China (24). Os resultados não podem ser comparados pelo facto de apenas existirem gráficos de evolução da temperatura.

Dos ensaios realizados no IPB, podemos concluir que o conjunto 9 é aquele que apresenta um menor fator de transmissão de calor, quando submetido a uma densidade de fluxo de calor de $5\left[\mathrm{~kW} / \mathrm{m}^{2}\right]$ e o conjunto 7 é 
aquele que apresenta melhor desempenho para um fluxo de calor de 20 $\left[\mathrm{kW} / \mathrm{m}^{2}\right]$.

O modelo numérico considera contacto perfeito entre camadas e não considera qualquer modelo de degradação térmica. No entanto, este modelo é capaz de prever a evolução da temperatura nos conjuntos submetidos a baixa densidade de fluxo de calor, em particular nas regiões de medição de T1, T2 e T3.

A análise paramétrica revelou que existe uma relação não linear entre o fluxo de calor incidente e o fluxo de calor transmitido. $O$ fator de transmissão de calor diminui com o aumento do fluxo incidente. Estas conclusões deverão ser verificadas com um maior número de ensaios experimentais a serem realizados, para maiores valores de densidade de fluxo de calor incidente e outros modelos de simulação.

\section{AGRADECIMENTOS}

Os autores agradecem o apoio da empresa LATINO GROUP na realização desta investigação.

\section{REFERÊNCIAS}

ATALAY, Ozgur; BAHADIR, Senem K.; KALAOGLU, Fatma. An Analysis on the Moisture and Thermal Protective Performance of Firefighter Clothing Based on Different Layer Combinations and Effect of Washing on Heat Protection and Vapour Transfer Performance. Advances in Materials Science and Engineering 2015.

CEN - European Committee for Standardization. EN ISO 13927 - Plastics Simple Heat Release Test Using a Conical Radiant Heater and a Thermopile Detector: 19, 2003. 
CEN - European Committee for Standardization. ISO 6942:2005. Protective Clothing. Protection against Heat and Fire. Method of Test: Evaluation of Materials and Material Assemblies When Exposed to a Source of Radiant Heat (ISO 6942:2002), NP EN ISO 6942:2005 [Portuguese Version]." : 18, 2005.

DAHAMNI, Salim; AGRED, Souhila; DOUADI, Hakim; BENAROUS, Abdallah. Numerical Prediction of Incoming Thermal Fluxes on a Firefighter Garment. In 2e Conférence Nationale Sur Les CFD et La Technologie, Draria, Algers, Algerria, 2018.

FU, M.; WENG, W.G.; YUAN, H.Y. Quantitative Assessment of the Relationship between Radiant Heat Exposure and Protective Performance of Multilayer Thermal Protective Clothing during Dry and Wet Conditions. Journal of Hazardous Materials 276: p.383-392, 2014.

GHAZY, Ahmed; BERGSTROM, Donald, J. Numerical Simulation of Heat Transfer in Firefighters' Protective Clothing with Multiple Air Gaps during Flash Fire Exposure. Numerical Heat Transfer; Part A: Applications 61(8): 569-593, 2012.

HUST, J. G.; LANKFORD, A. B.. Thermal Conductivity of $1 K$ To the Melting Point. National B. National Bureau of Standards. 1984.

KUTLU, Bengi; CIRELI, Aysun. Thermal Analysis and Performance Properties of Thermal Protective Clothing. Fibres and Textiles in Eastern Europe 13(3) p. 58-62, 2005.

LAWSON, James R.; WALTON, William D.; BRYNER, Nelson P.; AMON, Francine K. Estimates of Thermal Properties for Fire Fighters. Protective 
Clothing Materials. NISTIR 7282 (January): p.1-71, 2005.

LAWSON, James R.; MELL, William E.; PRASAD, Kuldeep. A Heat Transfer Model for Firefighters' Protective Clothing, Continued Developments in Protective Clothing Modeling. Fire Technology 46(1), p.833-41. 2010.

MELL, William E.; LAWSON, James R. A Heat Transfer Model for Firefighters ' Protective Clothing. Fire Technology 36(1), p. 833-841. 2000.

SONG, Guowen; WANG Faming, el al. Firefighters' Clothing and Equipment Firefighters' Clothing and Equipment: Performance, Protection and Comfort. CRC Press. eds. Guowen Song and Faming Wang, 2018.

STAGGS, J. E. J.; WHITELEY, R. H. Modelling the Combustion of SolidPhase Fuels in Cone Calorimeter Experiments. Fire and Materials 23(2), p.63-69, 1999.

STOLL, A. M.; CHIANTA, M. A. Heat Transfer through Fabrics as Related to Thermal Injury. Transactions of the New York Academy of Sciences 33(7) p.649-670, 1971.

SUN, G. H. S.; YOO, X. S.; ZHANG; PAN, N. Radiant Protective and Transport Properties of Fabrics Used by Wildland Firefighters. Textile Research Journal 70(7), p.567-573, 2000.

TIAN, Miao; WANG, Zhaoli; LI, Jun. 3D Numerical Simulation of Heat Transfer through Simplified Protective Clothing during Fire Exposure by CFD. International Journal of Heat and Mass Transfer 93: p.314-321, 2016. 